\title{
REGULARIZATION OF NONLINEAR ILL-POSED EQUATIONS WITH ACCRETIVE OPERATORS
}

\author{
YA. I. ALBER, C. E. CHIDUME, AND H. ZEGEYE
}

Received 11 October 2004

We study the regularization methods for solving equations with arbitrary accretive operators. We establish the strong convergence of these methods and their stability with respect to perturbations of operators and constraint sets in Banach spaces. Our research is motivated by the fact that the fixed point problems with nonexpansive mappings are namely reduced to such equations. Other important examples of applications are evolution equations and co-variational inequalities in Banach spaces.

\section{Introduction}

Let $E$ be a real normed linear space with dual $E^{*}$. The normalized duality mapping $j: E \rightarrow$ $2^{E^{*}}$ is defined by

$$
j(x):=\left\{x^{*} \in E^{*}:\left\langle x, x^{*}\right\rangle=\|x\|^{2},\left\|x^{*}\right\|_{*}=\|x\|\right\},
$$

where $\langle x, \phi\rangle$ denotes the dual product (pairing) between vectors $x \in E$ and $\phi \in E^{*}$. It is well known that if $E^{*}$ is strictly convex, then $j$ is single valued. We denote the single valued normalized duality mapping by $J$.

A map $A: D(A) \subseteq E \rightarrow 2^{E}$ is called accretive if for all $x, y \in D(A)$ there exists $J(x-y) \in$ $j(x-y)$ such that

$$
\langle u-v, J(x-y)\rangle \geq 0, \quad \forall u \in A x, \forall v \in A y .
$$

If $A$ is single valued, then (1.2) is replaced by

$$
\langle A x-A y, J(x-y)\rangle \geq 0 .
$$

$A$ is called uniformly accretive if for all $x, y \in D(A)$ there exist $J(x-y) \in j(x-y)$ and a strictly increasing function $\psi: \mathbb{R}^{+}:=[0, \infty) \rightarrow \mathbb{R}^{+}, \psi(0)=0$ such that

$$
\langle A x-A y, J(x-y)\rangle \geq \psi(\|x-y\|) .
$$


It is called strongly accretive if there exists a constant $k>0$ such that in (1.4) $\psi(t)=k t^{2}$. If $E$ is a Hilbert space, accretive operators are also called monotone. An accretive operator $A$ is said to be hemicontinuous at a point $x_{0} \in D(A)$ if the sequence $\left\{A\left(x_{0}+t_{n} x\right)\right\}$ converges weakly to $A x_{0}$ for any element $x$ such that $x_{0}+t_{n} x \in D(A), 0 \leq t_{n} \leq t\left(x_{0}\right)$ and $t_{n} \rightarrow 0$, $n \rightarrow \infty$. An accretive operator $A$ is said to be maximal accretive if it is accretive and the inclusion $G(A) \subseteq G(B)$, with $B$ accretive, where $G(A)$ and $G(B)$ denote graphs of $A$ and $B$, respectively, implies that $A=B$. It is known (see, e.g., [14]) that an accretive hemicontinuous operator $A: E \rightarrow E$ with a domain $D(A)=E$ is maximal accretive. In a smooth Banach space, a maximal accretive operator is strongly-weakly demiclosed on $D(A)$. An accretive operator $A$ is said to be $m$-accretive if $R(A+\alpha I)=E$ for all $\alpha>0$, where $I$ is the identity operator in $E$.

Interest in accretive maps stems mainly from their firm connection with fixed point problems, evolution equations and co-variational inequalites in a Banach space (see, e.g. $[6,7,8,9,10,11,12,26])$. Recall that each nonexpansive mapping is a continuous accretive operator $[7,19]$. It is known that many physically significant problems can be modeled by initial-value problems of the form (see, e.g., $[10,12,26]$ )

$$
x^{\prime}(t)+A x(t)=0, \quad x(0)=x_{0}
$$

where $A$ is an accretive operator in an appropriate Banach space. Typical examples where such evolution equations occur can be found in the heat, wave, or Schrödinger equations. One of the fundamental results in the theory of accretive operators, due to Browder [11], states that if $A$ is locally Lipschitzian and accretive, then $A$ is $m$-accretive. This result was subsequently generalized by Martin [23] to the continuous accretive operators. If $x(t)$ in (1.5) is independent of $t$, then (1.5) reduces to the equation

$$
A u=0,
$$

whose solutions correspond to the equilibrium points of the system (1.5). Consequently, considerable research efforts have been devoted, especially within the past 20 years or so, to iterative methods for approximating these equilibrium points.

The two well-known iterative schemes for successive approximation of a solution of the equation $A x=f$, where $A$ is either uniformly accretive or strongly accretive, are the Ishikawa iteration process (see, e.g., [20]) and the Mann iteration process (see, e.g., [22]). These iteration processes have been studied extensively by various authors and have been successfully employed to approximate solutions of several nonlinear operator equations in Banach spaces (see, e.g., $[13,15,17]$ ). But all efforts to use the Mann and the Ishikawa schemes to approximate the solution of the equation $A x=f$, where $A$ is an accretive-type mapping (not necessarily uniformly or strongly accretive), have not provided satisfactory results. The major obstacle is that for this class of operators the solution is not, in general, unique.

Our purpose in this paper is to construct approximations generated by regularization algorithms, which converge strongly to solutions of the equations $A x=f$ with accretive maps $A$ defined on subsets of Banach spaces. Our theorems are applicable to much larger classes of operator equations in uniformly smooth Banach spaces than previous results 
(see, e.g., [4]). Furthermore, the stability of our methods with respect to perturbation of the operators and constraint sets is also studied.

\section{Preliminaries}

Let $E$ be a real normed linear space of dimension greater than or equal to 2, and $x, y \in E$. The modulus of smoothness of $E$ is defined by

$$
\rho_{E}(\tau):=\sup \left\{\frac{\|x+y\|+\|x-y\|}{2}-1:\|x\|=1,\|y\|=\tau\right\} .
$$

A Banach space $E$ is called uniformly smooth if

$$
\lim _{\tau \rightarrow 0} h_{E}(\tau):=\lim _{\tau \rightarrow 0} \frac{\rho_{E}(\tau)}{\tau}=0 .
$$

Examples of uniformly smooth spaces are the Lebesgue $L_{p}$, the sequence $\ell_{p}$, and the Sobolev $W_{p}^{m}$ spaces for $1<p<\infty$ and $m \geq 1$ (see, e.g., [2]).

If $E$ is a real uniformly smooth Banach space, then the inequality

$$
\begin{aligned}
\|x\|^{2} & \leq\|y\|^{2}+2\langle x-y, J x\rangle \\
& \leq\|y\|^{2}+2\langle x-y, J y\rangle+2\langle x-y, J x-J y\rangle
\end{aligned}
$$

holds for every $x, y \in E$. A further estimation of $\|x\|^{2}$ needs one of the following two lemmas.

Lemma 2.1 [5]. Let E be a uniformly smooth Banach space. Then for $x, y \in E$,

$$
\langle x-y, J x-J y\rangle \leq 8\|x-y\|^{2}+C(\|x\|,\|y\|) \rho_{E}(\|x-y\|),
$$

where

$$
C(\|x\|,\|y\|) \leq 4 \max \{2 L,\|x\|+\|y\|\}
$$

and $L$ is the Figiel constant, $1<L<1.7[18,24]$.

LEMma 2.2 [2]. In a uniformly smooth Banach space E, for $x, y \in E$,

$$
\langle x-y, J x-J y\rangle \leq R^{2}(\|x\|,\|y\|) \rho_{E}\left(\frac{4\|x-y\|}{R(\|x\|,\|y\|)}\right),
$$

where

$$
R(\|x\|,\|y\|)=\sqrt{2^{-1}\left(\|x\|^{2}+\|y\|^{2}\right)} .
$$

If $\|x\| \leq R$ and $\|y\| \leq R$, then

$$
\langle x-y, J x-J y\rangle \leq 2 L R^{2} \rho_{E}\left(\frac{4\|x-y\|}{R}\right),
$$

where $L$ is the same as in Lemma 2.1. 
14 Nonlinear Ill-posed problems with accretive operators

We will need the following lemma on the recursive numerical inequalities.

Lemma 2.3 [1]. Let $\left\{\lambda_{k}\right\}$ and $\left\{\gamma_{k}\right\}$ be sequences of nonnegative numbers and let $\left\{\alpha_{k}\right\}$ be a sequence of positive numbers satisfying the conditions

$$
\sum_{1}^{\infty} \alpha_{n}=\infty, \quad \frac{\gamma_{n}}{\alpha_{n}} \longrightarrow 0 \quad \text { as } n \longrightarrow \infty
$$

Let the recursive inequality

$$
\lambda_{n+1} \leq \lambda_{n}-\alpha_{n} \phi\left(\lambda_{n}\right)+\gamma_{n}, \quad n=1,2, \ldots,
$$

be given where $\phi(\lambda)$ is a continuous and nondecreasing function from $\mathbb{R}^{+}$to $\mathbb{R}^{+}$such that it is positive on $\mathbb{R}^{+} \backslash\{0\}, \phi(0)=0, \lim _{t \rightarrow \infty} \phi(t) \geq c>0$. Then $\lambda_{n} \rightarrow 0$ as $n \rightarrow \infty$.

We will also use the concept of a sunny nonexpansive retraction [19].

Definition 2.4. Let $G$ be a nonempty closed convex subset of $E$. A mapping $Q_{G}: E \rightarrow G$ is said to be

(i) a retraction onto $G$ if $Q_{G}^{2}=Q_{G}$;

(ii) a nonexpansive retraction if it also satisfies the inequality

$$
\left\|Q_{G} x-Q_{G} y\right\| \leq\|x-y\|, \quad \forall x, y \in E ;
$$

(iii) a sunny retraction if for all $x \in E$ and for all $0 \leq t<\infty$,

$$
Q_{G}\left(Q_{G} x+t\left(x-Q_{G} x\right)\right)=Q_{G} x .
$$

Definition 2.5. If $Q_{G}$ satisfies (i)-(iii) of Definition 2.4, then the element $\tilde{x}=Q_{G} x$ is said to be a sunny nonexpansive retractor of $x \in E$ onto $G$.

Proposition 2.6. Let $E$ be a uniformly smooth Banach space, and let $G$ be a nonempty closed convex subset of $E$. A mapping $Q_{G}: E \rightarrow G$ is a sunny nonexpansive retraction if and only if for all $x \in E$ and for all $\xi \in G$,

$$
\left\langle x-Q_{G} x, J\left(Q_{G} x-\xi\right)\right\rangle \geq 0 .
$$

Denote by $\mathscr{H}_{E}\left(G_{1}, G_{2}\right)$ the Hausdorff distance between sets $G_{1}$ and $G_{2}$ in the space $E$, that is,

$$
\mathscr{H}_{E}\left(G_{1}, G_{2}\right)=\max \left\{\sup _{z_{1} \in G_{1}} \inf _{z_{2} \in G_{2}}\left\|z_{1}-z_{2}\right\|, \sup _{z_{1} \in G_{2}} \inf _{z_{2} \in G_{1}}\left\|z_{1}-z_{2}\right\|\right\} .
$$

Lemma 2.7 [7]. Let E be a uniformly smooth Banach space, and let $\Omega_{1}$ and $\Omega_{2}$ be closed convex subsets of E such that the Hausdorff distance $\mathcal{H}_{E}\left(\Omega_{1}, \Omega_{2}\right) \leq \sigma$. If $Q_{\Omega_{1}}$ and $Q_{\Omega_{2}}$ are the sunny nonexpansive retractions onto the subsets $\Omega_{1}$ and $\Omega_{2}$, respectively, then

$$
\left\|Q_{\Omega_{1}} x-Q_{\Omega_{2}} x\right\|^{2} \leq 16 R(2 r+q) h_{E}\left(16 L R^{-1} \sigma\right),
$$

where $h_{E}(\tau)=\tau^{-1} \rho_{E}(\tau)$, L is the Figiel constant, $r=\|x\|, q=\max \left\{q_{1}, q_{2}\right\}$, and $R=2(2 r+$ $q)+\sigma$. Here $q_{i}=\operatorname{dist}\left(\theta, \Omega_{i}\right), i=1,2$, and $\theta$ is the origin of the space $E$. 


\section{Operator regularization method}

We will deal with accretive operators $A: E \rightarrow E$ and operator equation

$$
A x=f
$$

given on a closed convex subset $G \subset D(A) \subseteq E$, where $D(A)$ is a domain of $A$.

In the sequel, we understand a solution of (3.1) in the sense of a solution of the covariational inequality (see, e.g., [9])

$$
\langle A x-f, J(y-x)\rangle \geq 0, \quad \forall y \in G, x \in G .
$$

The following statement is a motivation of this approach [25].

Theorem 3.1. Suppose that $E$ is a reflexive Banach space with strictly convex dual space $E^{*}$. Let $A: E \rightarrow E$ be a hemicontinuous operator. If for fixed $x^{*} \in E$ and $f \in E$ the co-variational inequality

$$
\left\langle A x-f, J\left(x-x^{*}\right)\right\rangle \geq 0, \quad \forall x \in E,
$$

holds, then $A x^{*}=f$.

In fact, the following more general theorem was proved in [8].

Theorem 3.2. Let $E$ be a smooth Banach space and let $A: E \rightarrow 2^{E}$ be an accretive operator. Then the following statements are equivalent:

(i) $x^{*}$ satisfies the covariational inequality

$$
\left\langle z-f, J\left(x-x^{*}\right)\right\rangle \geq 0, \quad \forall z \in A x, \forall x \in E
$$

(ii) $0 \in R\left(A x^{*}-f\right)$.

We present the following two definitions of a solution of the operator equation (3.1) on $G$.

Definition 3.3. An element $x^{*} \in G$ is said to be a generalized solution of the operator equation (3.1) on $G$ if there exists $z \in A x^{*}$ such that

$$
\left\langle z-f, J\left(y-x^{*}\right)\right\rangle \geq 0, \quad \forall y \in G
$$

Definition 3.4. An element $x^{*} \in G$ is said to be a total solution of the operator equation (3.1) on $G$ if

$$
\left\langle z-f, J\left(y-x^{*}\right)\right\rangle \geq 0, \quad \forall y \in G, \forall z \in A y .
$$

Lemma 3.5 [6]. Suppose that $E$ is a reflexive Banach space with strictly convex dual space $E^{*}$. Let $A$ be an accretive operator. If an element $x^{*} \in G$ is the generalized solution of (3.1) on $G$ characterized by the inequality (3.5), then it satisfies also the inequality (3.6), that is, it is a total solution of (3.1). 
Lemma 3.6 [6]. Suppose that $E$ is a reflexive Banach space with strictly convex dual space $E^{*}$. Let an operator $A$ be either hemicontinuous or maximal accretive. If $G \subset \operatorname{int} D(A)$, then Definitions 3.3 and 3.4 are equivalent.

LEMMA 3.7. Under the conditions of Lemma 3.6, the set of solutions of the operator equation (3.1) on $G$ is closed.

The proof follows from the fact that $J$ is continuous in smooth reflexive Banach spaces and any hemicontinuous or maximal accretive operator is demiclosed in such spaces.

For finding a solution $x^{*}$ of (3.1), we consider the regularized equation

$$
A z_{\alpha}+\alpha z_{\alpha}=f
$$

where $\alpha$ is a positive parameter.

Let $z_{\alpha}^{0}$ be a generalized solution of (3.7) on $G$, that is, there exists $\zeta_{\alpha}^{0} \in A z_{\alpha}^{0}$ such that

$$
\left\langle\zeta_{\alpha}^{0}+\alpha z_{\alpha}^{0}-f, J\left(x-z_{\alpha}^{0}\right)\right\rangle \geq 0, \quad \forall x \in G
$$

Theorem 3.8. Assume that $E$ is a reflexive Banach space with strictly convex dual space $E^{*}$ and with origin $\theta, A$ is a hemicontinuous or maximal accretive operator with domain $D(A) \subseteq E, G \subset \operatorname{int} D(A)$ is convex and closed, (3.1) has a nonempty generalized solution set $N \subset G$. Then $\left\|z_{\alpha}^{0}\right\| \leq 2\left\|\bar{x}^{*}\right\|$, where $\bar{x}^{*}$ is an element of $N$ with minimal norm. If the normalized duality mapping J is sequentially weakly continuous on $E$, then $z_{\alpha}^{0} \rightarrow \tilde{x}^{*}$ as $\alpha \rightarrow 0$, where $\tilde{x}^{*} \in N$ is a sunny nonexpansive retractor of $\theta$ onto $N$, that is, a (necessarily unique) solution of the inequality

$$
\left\langle\tilde{x}^{*}, J\left(x^{*}-\tilde{x}^{*}\right)\right\rangle \geq 0, \quad \forall x^{*} \in N \text {. }
$$

Proof. First, we show that $z_{\alpha}^{0}$ is the unique solution of (3.7). Suppose that $u_{\alpha}^{0}$ is another solution of this equation. Then along with (3.8), we have for some $\xi_{\alpha}^{0} \in A u_{\alpha}^{0}$ that

$$
\left\langle\xi_{\alpha}^{0}+\alpha u_{\alpha}^{0}-f, J\left(x-u_{\alpha}^{0}\right)\right\rangle \geq 0, \quad \forall x \in G
$$

Since $z_{\alpha}^{0} \in G$ and $u_{\alpha}^{0} \in G$, we have the inequalities

$$
\begin{gathered}
\left\langle\zeta_{\alpha}^{0}+\alpha z_{\alpha}^{0}-f, J\left(u_{\alpha}^{0}-z_{\alpha}^{0}\right)\right\rangle \geq 0, \\
\left\langle\xi_{\alpha}^{0}+\alpha u_{\alpha}^{0}-f, J\left(z_{\alpha}^{0}-u_{\alpha}^{0}\right)\right\rangle \geq 0 .
\end{gathered}
$$

Summing these inequalities, we obtain

$$
0 \geq\left\langle\xi_{\alpha}^{0}-\zeta_{\alpha}^{0}, J\left(z_{\alpha}^{0}-u_{\alpha}^{0}\right)\right\rangle \geq \alpha\left\langle z_{\alpha}^{0}-u_{\alpha}^{0}, J\left(z_{\alpha}^{0}-u_{\alpha}^{0}\right)\right\rangle=\alpha\left\|z_{\alpha}^{0}-u_{\alpha}^{0}\right\|^{2} .
$$

From this the claim follows.

Next, we prove that the sequence $\left\{z_{\alpha}^{0}\right\}$ is bounded. Observe that the covariational inequality (3.8) implies that

$$
\left\langle\zeta_{\alpha}^{0}+\alpha z_{\alpha}^{0}-f, J\left(x^{*}-z_{\alpha}^{0}\right)\right\rangle \geq 0, \quad \forall x^{*} \in N
$$


because $x^{*} \in G$. At the same time, since $x^{*}$ is a generalized solution of (3.1), there exists $\xi^{*} \in A x^{*}$ such that

$$
\left\langle\xi^{*}-f, J\left(z_{\alpha}^{0}-x^{*}\right)\right\rangle \geq 0
$$

Then (3.13) and (3.14) together give

$$
\left\langle\zeta_{\alpha}^{0}-\xi^{*}+\alpha z_{\alpha}^{0}, J\left(x^{*}-z_{\alpha}^{0}\right)\right\rangle=\left\langle\zeta_{\alpha}^{0}-\xi^{*}, J\left(x^{*}-z_{\alpha}^{0}\right)\right\rangle+\alpha\left\langle z_{\alpha}^{0}, J\left(x^{*}-z_{\alpha}^{0}\right)\right\rangle \geq 0 .
$$

By accretiveness of $A$, one gets

$$
\left\langle z_{\alpha}^{0}, J\left(x^{*}-z_{\alpha}^{0}\right)\right\rangle \geq 0
$$

The obtained inequality yields the estimates

$$
\left\|x^{*}-z_{\alpha}^{0}\right\|^{2} \leq\left\langle x^{*}, J\left(x^{*}-z_{\alpha}^{0}\right)\right\rangle \leq\left\|x^{*}\right\|\left\|x^{*}-z_{\alpha}^{0}\right\| .
$$

Hence, $\left\|z_{\alpha}^{0}\right\| \leq 2\left\|x^{*}\right\|$ for all $x^{*} \in N$, that is, $\left\|z_{\alpha}^{0}\right\| \leq 2\left\|\bar{x}^{*}\right\|$. Note that $\bar{x}^{*}$ exists because $N$ is closed and $E$ is reflexive.

Show now that $\left\|z_{\alpha}^{0}-\tilde{x}^{*}\right\| \rightarrow 0$ as $\alpha \rightarrow 0$. Since $\left\{z_{\alpha}^{0}\right\}$ is bounded, there exist a subsequence $z_{\beta}^{0} \subset z_{\alpha}^{0}$ and an element $\tilde{x} \in E$ such that $z_{\beta}^{0} \rightarrow \tilde{x}$ as $\beta \rightarrow 0$. Since $z_{\beta}^{0} \in G$ and $G$ is weakly closed (since it is closed and convex), we conclude that $\tilde{x} \in G$. Due to Lemma 3.6, the inequality (3.8) is equivalent to the following one:

$$
\left\langle w+\alpha x-f, J\left(x-z_{\alpha}^{0}\right)\right\rangle \geq 0, \quad \forall x \in G, \forall w \in A x .
$$

Therefore

$$
\left\langle w+\beta x-f, J\left(x-z_{\beta}^{0}\right)\right\rangle \geq 0, \quad \forall x \in G, \forall w \in A x .
$$

Passing to the limit in (3.19) as $\beta \rightarrow 0$ and using the weak continuity of $J$, one gets

$$
\langle w-f, J(x-\tilde{x})\rangle \geq 0, \quad \forall x \in G, \forall w \in A x .
$$

By Lemma 3.6 again, it follows that $\tilde{x}$ is a total (consequently, generalized) solution of (3.1) on $G$.

We now show that $\tilde{x}=\tilde{x}^{*}=Q_{N} \theta$ and $\tilde{x}^{*}$ is unique. This will mean that $z_{\alpha}^{0} \rightarrow \tilde{x}^{*}$ as we presumed above. Consider (3.17) on $\left\{z_{\beta}^{0}\right\}$ with $x^{*}=\tilde{x}$. It is clear that $\left\|\tilde{x}-z_{\beta}^{0}\right\| \rightarrow 0$. Then we deduce from (3.16) that

$$
\left\langle\tilde{x}, J\left(x^{*}-\tilde{x}\right)\right\rangle \geq 0, \quad \forall x^{*} \in N
$$

This means that $\tilde{x}=Q_{N} \theta$.

We prove that $\tilde{x}$ is a unique solution of the last inequality. Suppose that $\tilde{x}_{1} \in N$ is its another solution. Then

$$
\left\langle\tilde{x}_{1}, J\left(x^{*}-\tilde{x}_{1}\right)\right\rangle \geq 0, \quad \forall x^{*} \in N .
$$


18 Nonlinear Ill-posed problems with accretive operators

We have

$$
\begin{gathered}
\left\langle\tilde{x}, J\left(\tilde{x}_{1}-\tilde{x}\right)\right\rangle \geq 0, \\
\left\langle\tilde{x}_{1}, J\left(\tilde{x}-\tilde{x}_{1}\right)\right\rangle \geq 0 .
\end{gathered}
$$

Their combination gives

$$
\left\langle\tilde{x}-\tilde{x}_{1}, J\left(\tilde{x}_{1}-\tilde{x}\right)\right\rangle \geq 0
$$

which contradicts the fact that $\left\|\tilde{x}-\tilde{x}_{1}\right\| \geq 0$. Thus, the claim is true.

Finally, the first inequality in (3.17) implies the strong convergence of $\left\{z_{\alpha}^{0}\right\}$ to $\bar{x}^{*}$. The proof is accomplished. In particular, the theorem is valid if $N$ is a singleton.

Next we will study an operator regularization method for (3.1) with a perturbed righthand side, perturbed constraint set, and perturbed operator. Assume that, instead of $f$, $G$, and $A$, we have the sequences $\left\{f^{\delta}\right\} \in E,\left\{G_{\sigma}\right\} \in E$, and $\left\{A^{\omega}\right\}, A^{\omega}: G_{\sigma} \rightarrow E$, such that

$$
\begin{gathered}
\left\|f^{\delta}-f\right\| \leq \delta, \\
\mathcal{H}_{E}\left(G_{\sigma}, G\right) \leq \sigma,
\end{gathered}
$$

where $\mathscr{H}_{E}\left(G_{1}, G_{2}\right)$ is the Hausdorff distance (2.14), and

$$
\left\|A^{\omega} x-A x\right\| \leq \omega \zeta(\|x\|), \quad \forall x \in D
$$

where $\zeta(t)$ is a positive and bounded function defined on $\mathbb{R}^{+}$and $D=D(A)=D\left(A^{\omega}\right)$. Thus, in reality, the equations

$$
A^{\omega} y=f^{\delta}
$$

are given on $G_{\sigma}, \sigma \geq 0$. Consider the following regularized equation on $G_{\sigma}$ :

$$
A^{\omega} z+\alpha z=f^{\delta}
$$

Let $z_{\alpha}^{\gamma}$ with $\gamma=(\delta, \sigma, \omega)$ be its (unique) generalized solution. This means that there exists $y_{\alpha}^{\gamma} \in A^{\omega} z_{\alpha}^{\gamma}$ such that

$$
\left\langle y_{\alpha}^{\gamma}+\alpha z_{\alpha}^{\gamma}-f^{\delta}, J\left(x-z_{\alpha}^{\gamma}\right)\right\rangle \geq 0, \quad \forall x \in G_{\sigma} .
$$

Theorem 3.9. Assume that

(i) in real uniformly smooth Banach space $E$ with the modulus of smoothness $\rho_{E}(\tau)$, all the conditions of Theorem 3.8 are fulfilled;

(ii) (3.28) has bounded generalized solutions $z_{\alpha}^{\gamma}$ for all $\delta \geq 0, \sigma \geq 0, \omega \geq 0$, and $\alpha>0$;

(iii) the operators $A^{\omega}$ are accretive and bounded (i.e., they carry bounded sets of $E$ to bounded sets of $E$ );

(iv) $G \subset D$ and $G_{\sigma} \subset D$ are convex and closed sets;

(v) the estimates (3.25) and (3.26) are satisfied for $\delta \geq 0, \sigma \geq 0$, and $\omega \geq 0$. 


$$
\frac{\delta+\omega+h_{E}(\sigma)}{\alpha} \longrightarrow 0 \quad \text { as } \alpha \longrightarrow 0
$$

then $z_{\alpha}^{\gamma} \rightarrow \bar{x}^{*}$, where $\bar{x}^{*}$ is a sunny nonexpansive retractor of $\theta$ onto $N$.

Proof. Write the obvious inequality

$$
\left\|z_{\alpha}^{\gamma}-\bar{x}^{*}\right\| \leq\left\|z_{\alpha}^{0}-\bar{x}^{*}\right\|+\left\|z_{\alpha}^{\gamma}-z_{\alpha}^{0}\right\|
$$

where $z_{\alpha}^{0}$ is a generalized solution of (3.7). The limit relation $\left\|z_{\alpha}^{0}-\bar{x}^{*}\right\| \rightarrow 0$ has been already established in Theorem 3.8. At the same time, the result $\left\|z_{\alpha}^{\gamma}-z_{\alpha}^{0}\right\| \rightarrow 0$ immediately follows from Lemma 4.1 proved in the next section. The condition (3.30) is sufficient for this conclusion.

Remark 3.10. We do not suppose that in the operator equation (3.28) every operator $A^{\omega}$ has been defined on every set $G_{\sigma}$. Only possibility for the parameters $\omega$ and $\sigma$ to be simultaneously rushed to zero is required.

\section{Proximity lemma}

We further present the proximity lemma between solutions of two regularized equations

$$
\begin{array}{ll}
T_{1} z_{1}+\alpha_{1} z_{1}=f_{1}, & \alpha_{1}>0, \\
T_{2} z_{2}+\alpha_{2} z_{2}=f_{2}, & \alpha_{2}>0,
\end{array}
$$

on $G_{1}$ and $G_{2}$, respectively, provided their intersection $G_{1} \cap G_{2}$ is not empty.

Lemma 4.1 (cf. [3]). Suppose that

(i) $E$ is a real uniformly smooth Banach space with the modulus of smoothness $\rho_{E}(\tau)$;

(ii) the solution sequences $\left\{z_{1}\right\}$ and $\left\{z_{2}\right\}$ of (4.1) and (4.2), respectively, are bounded, that is, there exists a constant $M_{1}>0$ such that $\left\|z_{1}\right\| \leq M_{1}$ and $\left\|z_{2}\right\| \leq M_{1}$;

(iii) the operators $T_{1}$ and $T_{2}$ are accretive and bounded on the sequences $\left\{z_{1}\right\}$ and $\left\{z_{2}\right\}$, that is, there exist constants $M_{2}>0$ and $M_{3}>0$ such that $\left\|T_{1} z_{1}\right\| \leq M_{2}$ and $\left\|T_{2} z_{2}\right\| \leq$ $M_{3}$;

(iv) $G_{1} \subset D$ and $G_{2} \subset D$ are convex and closed subsets of $E$ and $D=D\left(T_{1}\right)=D\left(T_{2}\right)$;

(v) the estimates $\left\|f_{1}-f_{2}\right\| \leq \delta, \mathcal{H}_{E}\left(G_{1}, G_{2}\right) \leq \sigma$, and $\left\|T_{1} z-T_{2} z\right\| \leq \omega \zeta(\|z\|), \forall z \in D$, are fulfilled. Then

$$
\left\|z_{1}-z_{2}\right\| \leq \zeta\left(M_{1}\right) \frac{\omega}{\alpha_{1}}+\frac{\delta}{\alpha_{1}}+M_{1} \frac{\left|\alpha_{1}-\alpha_{2}\right|}{\alpha_{1}}+\sqrt{\frac{c_{1} h_{E}\left(c_{2} \sigma\right)}{\alpha_{1}}}
$$

where

$$
c_{1}=8 R\left(2 \alpha_{1} M_{1}+M_{2}+M_{3}+\left\|f_{1}\right\|+\left\|f_{2}\right\|\right), \quad c_{2}=16 L R^{-1}, \quad R=2 M_{1}+\sigma .
$$


20 Nonlinear Ill-posed problems with accretive operators

Proof. Solutions $z_{1} \in G_{1}$ and $z_{2} \in G_{2}$ of the operator equations (4.1) and (4.2) are defined by the following co-variational inequalities, respectively:

$$
\begin{aligned}
& \left\langle T_{1} z_{1}+\alpha_{1} z_{1}-f_{1}, J\left(x-z_{1}\right)\right\rangle \geq 0, \quad \forall x \in G_{1}, \alpha_{1}>0, \\
& \left\langle T_{2} z_{2}+\alpha_{2} z_{2}-f_{2}, J\left(x-z_{2}\right)\right\rangle \geq 0, \quad \forall x \in G_{2}, \alpha_{2}>0 .
\end{aligned}
$$

Estimate a dual product

$$
B=\left\langle T_{1} z_{1}+\alpha_{1} z_{1}-f_{1}-T_{2} z_{2}-\alpha_{2} z_{2}+f_{2}, J\left(z_{1}-z_{2}\right)\right\rangle .
$$

Obviously,

$$
B=\left\langle T_{1} z_{1}-T_{1} z_{2}+\alpha_{1}\left(z_{1}-z_{2}\right)+T_{1} z_{2}-T_{2} z_{2}+\left(\alpha_{1}-\alpha_{2}\right) z_{2}+f_{2}-f_{1}, J\left(z_{1}-z_{2}\right)\right\rangle .
$$

The operator $T_{1}$ is accretive, therefore,

$$
\left\langle T_{1} z_{1}-T_{1} z_{2}, J\left(z_{1}-z_{2}\right)\right\rangle \geq 0 .
$$

Then

$$
B \geq \alpha_{1}|| z_{1}-z_{2}\left\|^{2}-\left(\left\|T_{1} z_{2}-T_{2} z_{2}\right\|+\left|\alpha_{1}-\alpha_{2}\right|\left\|z_{2}\right\|+\left\|f_{1}-f_{2}\right\|\right)\right\| z_{1}-z_{2} \| .
$$

Since $\left\|z_{2}\right\| \leq M_{1}$, we conclude in conformity with (v) that

$$
B \geq-c\left\|z_{1}-z_{2}\right\|+\alpha_{1}\left\|z_{1}-z_{2}\right\|^{2}
$$

where

$$
c=\omega \zeta\left(M_{1}\right)+M_{1}\left|\alpha_{1}-\alpha_{2}\right|+\delta .
$$

Next, if $\mathscr{H}_{E}\left(G_{1}, G_{2}\right) \leq \sigma$, then for every $z_{2} \in G_{2}$ there exists $\tilde{z} \in G_{1}$ such that $\left\|z_{2}-\tilde{z}\right\| \leq \sigma$ and

$$
\begin{aligned}
\left\langle T_{1} z_{1}+\right. & \left.\alpha_{1} z_{1}-f_{1}, J\left(z_{1}-z_{2}\right)\right\rangle \\
= & \left\langle T_{1} z_{1}+\alpha_{1} z_{1}-f_{1}, J\left(z_{1}-z_{2}\right)+J\left(z_{1}-\tilde{z}\right)-J\left(z_{1}-\tilde{z}\right)\right\rangle \\
= & \left\langle T_{1} z_{1}+\alpha_{1} z_{1}-f_{1}, J\left(z_{1}-\tilde{z}\right)\right\rangle \\
& +\left\langle T_{1} z_{1}+\alpha_{1} z_{1}-f_{1}, J\left(z_{1}-z_{2}\right)-J\left(z_{1}-\tilde{z}\right)\right\rangle .
\end{aligned}
$$

By (4.5),

$$
\left\langle T_{1} z_{1}+\alpha_{1} z_{1}-f_{1}, J\left(z_{1}-\tilde{z}\right)\right\rangle \leq 0 .
$$

Estimate the last term in (4.13). For this recall that if $\|x\| \leq R$ and $\|y\| \leq R$, then (see [2, page 38])

$$
\|J(x)-J(y)\|_{*} \leq 8 R h_{E}\left(16 L R^{-1}\|x-y\|\right)
$$


For $\left\|z_{1}-z_{2}\right\| \leq 2 M_{1}$ and $\left\|z_{1}-\tilde{z}\right\| \leq 2 M_{1}+\sigma=R$, this implies that

$$
\begin{aligned}
\left\langle T_{1} z_{1}\right. & \left.+\alpha_{1} z_{1}-f_{1}, J\left(z_{1}-z_{2}\right)-J\left(z_{1}-\tilde{z}\right)\right\rangle \\
& \leq\left\|T_{1} z_{1}+\alpha_{1} z_{1}-f_{1}\right\|\left\|J\left(z_{1}-z_{2}\right)-J\left(z_{1}-\tilde{z}\right)\right\|_{*} \\
& \leq 8 R\left(\alpha_{1}\left\|z_{1}\right\|+\left\|T_{1} z_{1}\right\|+\left\|f_{1}\right\|\right) h_{E}\left(16 L R^{-1}\left\|z_{2}-\tilde{z}\right\|\right) \\
& \leq 8 R\left(\alpha_{1} M_{1}+M_{2}+\left\|f_{1}\right\|\right) h_{E}\left(16 L R^{-1} \sigma\right) .
\end{aligned}
$$

Analogously to the previous chain of inequalities,

$$
\left\langle T_{2} z_{2}+\alpha_{2} z_{2}-f_{2}, J\left(z_{2}-z_{1}\right)\right\rangle \leq 8 R\left(\alpha_{1} M_{1}+M_{3}+\left\|f_{2}\right\|\right) h_{E}\left(16 L R^{-1} \sigma\right) .
$$

Therefore,

$$
B \leq c_{1} h_{E}\left(c_{2} \sigma\right)
$$

Finally, combining (4.11) with (4.18), one gets

$$
c_{1} h_{E}\left(c_{2} \sigma\right)+c\left\|z_{1}-z_{2}\right\| \geq \alpha_{1}\left\|z_{1}-z_{2}\right\|^{2}
$$

This quadratic inequality gives

$$
\left\|z_{1}-z_{2}\right\| \leq \frac{c+\sqrt{c^{2}+4 \alpha_{1} c_{1} h_{E}\left(c_{2} \sigma\right)}}{2 \alpha_{1}} \leq \frac{c}{\alpha_{1}}+\sqrt{\frac{c_{1} h_{E}\left(c_{2} \sigma\right)}{\alpha_{1}}}
$$

because $\sqrt{a+b} \leq \sqrt{a}+\sqrt{b}$ for all $a, b \geq 0$. Thus, (4.3) holds.

From Theorem (3.10) and Lemma 4.1 we obtain the following corollary.

Corollary 4.2. If, in the conditions of Lemma 4.1, $\omega=\delta=\sigma=0$, that is, $T_{1}=T_{2}, f_{1}=f_{2}$, and $G_{1}=G_{2}$, then

$$
\left\|z_{1}-z_{2}\right\| \leq 2|| x^{*} \| \frac{\left|\alpha_{1}-\alpha_{2}\right|}{\alpha_{1}}
$$

\section{Iterative regularization methods}

5.1. We begin by considering iterative regularization with exact given data.

THEOREM 5.1. Let E be a real uniformly smooth Banach space with the modulus of smoothness $\rho_{E}(\tau)$, let $A: E \rightarrow E$ be a bounded accretive operator with $D(A) \subseteq E$, and let $G \subset$ $\operatorname{int} D(A)$ be a closed convex set. Suppose that (3.1) has a generalized solution $x^{*}$ on $G$. Let $\left\{\epsilon_{n}\right\}$ and $\left\{\alpha_{n}\right\}$ be real sequences such that $\epsilon_{n} \leq 1, \alpha_{n} \leq 1$. Starting from arbitrary $x_{0} \in G$ define the sequence $\left\{x_{n}\right\}$ as follows:

$$
x_{n+1}:=Q_{G}\left(x_{n}-\epsilon_{n}\left(A x_{n}+\alpha_{n} x_{n}-f\right)\right), \quad n=0,1,2, \ldots,
$$




\section{Nonlinear Ill-posed problems with accretive operators}

where $Q_{G}$ is a nonexpansive retraction of $E$ onto $G$. Then there exists $1>d>0$ such that whenever

$$
\epsilon_{n} \leq d, \quad \frac{\rho_{E}\left(\epsilon_{n}\right)}{\epsilon_{n} \alpha_{n}} \leq d^{2}
$$

for all $n \geq 0$, the sequence $\left\{x_{n}\right\}$ is bounded.

Proof. Denote by $B_{r}\left(x^{*}\right)$ the closed ball of radius $r$ with the center in $x^{*}$. Choose $r>0$ sufficiently large such that $r \geq 2\left\|x^{*}\right\|$ and $x_{0} \in B_{r}\left(x^{*}\right)$. Construct the set $S=B_{r}\left(x^{*}\right) \cap G$ and let

$$
M:=\frac{3}{2} r+\|f\|+\sup \{\|A x\|: x \in S\} .
$$

We claim that $\left\{x_{n}\right\}$ is bounded in our circumstances. Show by induction that $x_{n} \in S$ for all positive integers. Actually, $x_{0} \in S$ by the assumption. Hence, for given $n>0$, we may presume the inclusion $x_{n} \in S$ and prove that $x_{n+1} \in S$. Suppose that $x_{n+1}$ does not belong to $S$. Since $x_{n+1} \in G$, this means that $\left\|x_{n+1}-x^{*}\right\|>r$. By (5.1) and due to the nonexpansiveness of $Q_{G}$, we have

$$
\begin{aligned}
\left\|x_{n+1}-x^{*}\right\| & =\left\|Q_{G}\left(x_{n}-\epsilon_{n}\left(A x_{n}+\alpha_{n} x_{n}-f\right)\right)-Q_{G} x^{*}\right\| \\
& \leq\left\|x_{n}-x^{*}-\epsilon_{n}\left(A x_{n}+\alpha_{n} x_{n}-f\right)\right\| \\
& \leq\left\|x_{n}-x^{*}\right\|+\epsilon_{n}\left\|A x_{n}+\alpha_{n} x_{n}-f\right\| \\
& \leq\left\|x_{n}-x^{*}\right\|+\left\|A x_{n}\right\|+\left\|x_{n}-x^{*}\right\|+\left\|x^{*}\right\|+\|f\| \\
& \leq r+\sup _{x \in S}\|A x\|+r+\frac{1}{2} r+\|f\|=r+M=\bar{M} .
\end{aligned}
$$

In the next calculations, we apply Lemma 2.2 with $x=x_{n+1}-x^{*}$ and $y=x_{n}-x^{*}$. It is easy to see that

$$
\begin{gathered}
\|x\|=\left\|x_{n+1}-x^{*}\right\| \leq \bar{M}, \quad\|y\|=\left\|x_{n}-x^{*}\right\| \leq r, \\
\|x-y\|=\left\|x_{n+1}-x_{n}\right\| \leq \varepsilon_{n}\left\|A x_{n}+\alpha_{n} x_{n}-f\right\| \leq \varepsilon_{n} M .
\end{gathered}
$$

Thus, $\max \{\|x\|,\|y\|\} \leq \bar{M}$, and we have

$$
\left\langle x_{n+1}-x_{n}, J\left(x_{n+1}-x^{*}\right)-J\left(x_{n}-x^{*}\right)\right\rangle \leq 2 L \bar{M}^{2} \rho_{E}\left(4 M \bar{M}^{-1} \epsilon_{n}\right),
$$

because the function $\rho_{E}(\tau)$ is nondecreasing $[18,21]$. Besides, the function $\rho_{E}(\tau)$ is convex, therefore, $\rho_{E}(c \tau) \leq c \rho_{E}(\tau)$, for all $c \leq 1$. Since $M \bar{M}^{-1} \leq 1$, (5.6) yields

$$
\left\langle x_{n+1}-x_{n}, J\left(x_{n+1}-x^{*}\right)-J\left(x_{n}-x^{*}\right)\right\rangle \leq 2 L M \bar{M} \rho_{E}\left(4 \varepsilon_{n}\right) .
$$

Then using the facts that $\rho_{E}(\tau)$ is continuous, $0 \leq \varepsilon_{n} \leq 1$, and by [16],

$$
2 \leq \lim _{\tau \rightarrow 0} \frac{\rho_{E}(4 \tau)}{\rho_{E}(2 \tau)} \leq 4
$$


we conclude that there is a constant $C>1$ such that

$$
\left\langle x_{n+1}-x_{n}, J\left(x_{n+1}-x^{*}\right)-J\left(x_{n}-x^{*}\right)\right\rangle \leq 8 \operatorname{LCM} \bar{M} \rho_{E}\left(\varepsilon_{n}\right) .
$$

Moreover, by (2.3), (5.1), (5.6) and by the inclusion $x_{n} \in S$, one gets

$$
\begin{aligned}
\left\|x_{n+1}-x^{*}\right\|^{2} \leq & \left\|x_{n}-x^{*}-\epsilon_{n}\left(A x_{n}+\alpha_{n} x_{n}-f\right)\right\|^{2} \\
\leq & \left\|x_{n}-x^{*}\right\|^{2}-2 \epsilon_{n}\left\langle A x_{n}-f, J\left(x_{n}-x^{*}\right)\right\rangle \\
& -2 \epsilon_{n} \alpha_{n}\left\langle x_{n}, J\left(x_{n}-x^{*}\right)\right\rangle+16 \operatorname{LCM} \bar{M} \rho_{E}\left(\varepsilon_{n}\right) .
\end{aligned}
$$

Since $x^{*}$ is a generalized solution of (3.1) on $G$ and $x_{n} \in G$ for all $n \geq 0$, we can write

$$
\left\langle A x_{n}-f, J\left(x_{n}-x^{*}\right)\right\rangle \geq 0 .
$$

Then (5.10) implies the inequality

$$
\left\|x_{n+1}-x^{*}\right\|^{2} \leq\left\|x_{n}-x^{*}\right\|^{2}-2 \epsilon_{n} \alpha_{n}\left\langle x_{n}, J\left(x_{n}-x^{*}\right)\right\rangle+16 L C \bar{M} \rho_{E}\left(\varepsilon_{n}\right) .
$$

Choose $K>0$ such that

$$
K \leq \frac{r^{2}}{4(\sqrt{D}+M)^{2}}
$$

where $D=8 L C M \bar{M}$. Set $d:=\sqrt{K}$. It is not difficult to verify that $1>d>0$. By virtue of our assumption, $\left\|x_{n+1}-x^{*}\right\|>\left\|x_{n}-x^{*}\right\|$. This allows us to deduce from (5.12) the following estimate:

$$
\epsilon_{n} \alpha_{n}\left\langle x_{n}, J\left(x_{n}-x^{*}\right)\right\rangle \leq 8 \operatorname{LCM} \bar{M} \rho_{E}\left(\epsilon_{n}\right) .
$$

It gives the inequality

$$
\left\langle x_{n}, J\left(x_{n}-x^{*}\right)\right\rangle \leq D K
$$

because of the assumption that

$$
\frac{\rho_{E}\left(\epsilon_{n}\right)}{\alpha_{n} \epsilon_{n}} \leq K=d^{2}, \quad \forall n \geq 0 .
$$

Now adding $\left\langle-x^{*}, J\left(x_{n}-x^{*}\right)\right\rangle$ to both sides of (5.15), we get

$$
\begin{aligned}
\left\|x_{n}-x^{*}\right\|^{2} & \leq K D+\left\langle-x^{*}, J\left(x_{n}-x^{*}\right)\right\rangle \\
& \leq K D+\left\|x^{*}\right\|\left\|x_{n}-x^{*}\right\| \leq K D+\frac{r}{2}\left\|x_{n}-x^{*}\right\| .
\end{aligned}
$$

Solving this quadratic inequality for $\left\|x_{n}-x^{*}\right\|$ and using the estimate

$$
\sqrt{\frac{r^{2}}{16}+K D} \leq \frac{r}{4}+\sqrt{K D},
$$


24 Nonlinear Ill-posed problems with accretive operators

we derive that

$$
\left\|x_{n}-x^{*}\right\| \leq \frac{r}{2}+\sqrt{K D}
$$

In any case,

$$
\left\|x_{n+1}-x^{*}\right\| \leq\left\|x_{n}-x^{*}\right\|+\epsilon_{n}\left\|A x_{n}+\alpha_{n} x_{n}-f\right\|,
$$

so that

$$
\left\|x_{n+1}-x^{*}\right\| \leq \frac{r}{2}+\sqrt{K D}+\epsilon_{n} M<r
$$

by the original choices of $K$ and $\epsilon_{n}$, and this contradicts the assumption that $x_{n+1}$ is not in $S$. Therefore $x_{n} \in S$ for any integers $n \geq 0$. Thus $\left\{x_{n}\right\}$ is bounded, say, $\left\|x_{n}\right\| \leq \widetilde{C}$.

In what follows, we suppose that the normalized duality mapping $J$ is continuous and sequentially weakly continuous in the ball $B_{r}(\theta)$ with $r=\widetilde{C}$. We show that $x_{n} \rightarrow \bar{x}^{*}$, where $\bar{x}^{*}$ is a unique solution of (3.9).

Theorem 5.2. Assume that all the conditions of Theorems 3.8 and 5.1 are fulfilled. In addition, let $\alpha_{n} \rightarrow 0$ as $n \rightarrow \infty$,

$$
\frac{\epsilon_{n}}{\alpha_{n}} \longrightarrow 0, \quad \frac{\left|\alpha_{n}-\alpha_{n+1}\right|}{\epsilon_{n} \alpha_{n}^{2}} \longrightarrow 0, \quad \frac{\rho_{E}\left(\epsilon_{n}\right)}{\epsilon_{n} \alpha_{n}} \longrightarrow 0 .
$$

Then the sequence $\left\{x_{n}\right\}$ generated by (5.1) converges strongly to $\bar{x}^{*}$ as $n \rightarrow \infty$.

Proof. So, by Theorem 5.1, $\left\{x_{n}\right\}$ is bounded by a constant $\widetilde{C}$. Let $z_{n}$ and $z_{n+1}$ be generalized solutions of the equation

$$
A z+\alpha_{k} z=f
$$

on $G$ for $k=n$ and $k=n+1$, respectively. It follows from (4.3) and (5.22) that there exists a constant $d>0$ such that $\left\|z_{n}-z_{n+1}\right\| \leq d$. Put

$$
p_{n}=x_{n}-\epsilon_{n}\left(A x_{n}+\alpha_{n} x_{n}-f\right)
$$

Then by (5.1) and by convexity of the functional $\|x\|^{2}$, we have that

$$
\begin{aligned}
\left\|x_{n+1}-z_{n+1}\right\|^{2}= & \left\|Q_{G} p_{n}-Q_{G} z_{n+1}\right\|^{2} \\
\leq & \left\|p_{n}-z_{n+1}\right\|^{2} \\
\leq & \left\|p_{n}-z_{n}\right\|^{2}+2\left\langle z_{n+1}-z_{n}, J\left(z_{n+1}-p_{n}\right)\right\rangle \\
\leq & \left\|p_{n}-z_{n}\right\|^{2}+2\left\langle z_{n+1}-z_{n}, J\left(z_{n}-p_{n}\right)\right\rangle \\
& +2\left\langle z_{n+1}-z_{n}, J\left(z_{n+1}-p_{n}\right)-J\left(z_{n}-p_{n}\right)\right\rangle .
\end{aligned}
$$


We continue the estimation of (5.25) using Lemma 2.1. It is easy to see that if $H$ is a Hilbert space and $\tau \leq \bar{\tau}$, then $[18,21]$

$$
\rho_{E}(\tau) \geq \rho_{H}(\tau)=\left(1+\tau^{2}\right)^{1 / 2}-1 \geq \bar{c} \tau^{2}
$$

where

$$
\bar{c}=\left(\sqrt{1+\bar{\tau}^{2}}+1\right)^{-1} .
$$

One gets

$$
\begin{aligned}
\left\|x_{n+1}-z_{n+1}\right\|^{2} \leq & \left\|p_{n}-z_{n}\right\|^{2}+2\left\|z_{n}-p_{n}\right\| \cdot\left\|z_{n+1}-z_{n}\right\| \\
& +16\left\|z_{n+1}-z_{n}\right\|^{2}+C_{1}(n) \rho_{E}\left(\left\|z_{n+1}-z_{n}\right\|\right),
\end{aligned}
$$

where

$$
\begin{aligned}
C_{1}(n) & =8 \max \left\{2 L,\left\|z_{n}-p_{n}\right\|+\left\|z_{n+1}-p_{n}\right\|\right\} \\
& \leq 8 \max \left\{2 L, \widetilde{C}+M+2\left\|x^{*}\right\|\right\}=C_{1},
\end{aligned}
$$

where $M$ is defined by (5.3). Therefore, due to Corollary 4.2,

$$
\begin{aligned}
\left\|x_{n+1}-z_{n+1}\right\|^{2} \leq & \left\|p_{n}-z_{n}\right\|^{2}+4 \frac{\left|\alpha_{n}-\alpha_{n+1}\right|}{\alpha_{n}} \cdot\left\|x^{*}\right\| \cdot\left\|p_{n}-z_{n}\right\| \\
& +\left(16 \bar{c}^{-1}+C_{1}\right) \rho_{E}\left(\frac{2\left(\alpha_{n}-\alpha_{n+1}\right)}{\alpha_{n}}\left\|x^{*}\right\|\right),
\end{aligned}
$$

and in (5.27) $\bar{\tau}=d \geq\left\|z_{n+1}-z_{n}\right\|=\tau$.

Now we evaluate $\left\|p_{n}-z_{n}\right\|^{2}$. The convexity inequality (2.3) yields

$$
\begin{aligned}
\left\|p_{n}-z_{n}\right\|^{2}= & \left\|x_{n}-\epsilon_{n}\left(A x_{n}+\alpha_{n} x_{n}-f\right)-z_{n}\right\|^{2} \\
\leq & \left\|x_{n}-z_{n}\right\|^{2}-2 \epsilon_{n}\left\langle A x_{n}+\alpha_{n} x_{n}-f, J\left(p_{n}-z_{n}\right)\right\rangle \\
= & \left\|x_{n}-z_{n}\right\|^{2}-2 \epsilon_{n}\left\langle A x_{n}+\alpha_{n} x_{n}-f, J\left(x_{n}-z_{n}\right)\right\rangle \\
& +2\left\langle p_{n}-x_{n}, J\left(p_{n}-z_{n}\right)-J\left(x_{n}-z_{n}\right)\right\rangle .
\end{aligned}
$$

Since

$$
\left\langle A z_{n}+\alpha_{n} z_{n}-f, J\left(x_{n}-z_{n}\right)\right\rangle \geq 0,
$$

and by the accretiveness property of $A$,

$$
\left\langle A x_{n}-A z_{n}, J\left(x_{n}-z_{n}\right)\right\rangle \geq 0,
$$


we deduce

$$
\begin{aligned}
\left\|p_{n}-z_{n}\right\|^{2} \leq & \left\|x_{n}-z_{n}\right\|^{2}-2 \epsilon_{n} \alpha_{n}\left\|x_{n}-z_{n}\right\|^{2} \\
& +2\left\langle p_{n}-x_{n}, J\left(p_{n}-z_{n}\right)-J\left(x_{n}-z_{n}\right)\right\rangle \\
\leq & \left\|x_{n}-z_{n}\right\|^{2}-2 \epsilon_{n} \alpha_{n}\left\|x_{n}-z_{n}\right\|^{2}+16 \epsilon_{n}^{2}\left\|A x_{n}+\alpha_{n} x_{n}-f\right\|^{2} \\
& +C_{2}(n) \rho_{E}\left(\epsilon_{n}\left\|A x_{n}+\alpha_{n} x_{n}-f\right\|\right),
\end{aligned}
$$

where

$$
\begin{aligned}
C_{2}(n) & =4 \max \left\{2 L,\left\|z_{n}-p_{n}\right\|+\left\|x_{n}-z_{n}\right\|\right\} \\
& \leq 4 \max \left\{2 L, 2 \widetilde{C}+M+2\left\|x^{*}\right\|\right\}=C_{2} .
\end{aligned}
$$

Substituting (5.34) for (5.30) and using the fact that $\rho_{E}(\tau) \leq \tau$, we obtain

$$
\begin{aligned}
\left\|x_{n+1}-z_{n+1}\right\|^{2} \leq & \left\|x_{n}-z_{n}\right\|^{2}-2 \epsilon_{n} \alpha_{n}\left\|x_{n}-z_{n}\right\|^{2}+16 \epsilon_{n}^{2} M^{2} \\
& \left.+C_{2} \rho_{E}\left(\epsilon_{n} M\right)+C_{3} \frac{\left|\alpha_{n}-\alpha_{n+1}\right|}{\alpha_{n}}, C_{1}\right) \rho_{E}\left(\frac{\left(\alpha_{n}-\alpha_{n+1}\right)}{\alpha_{n}} M\right),
\end{aligned}
$$

where

$$
C_{3}=M C_{1}+4\left\|x^{*}\right\|\left(\widetilde{C}+M+2\left\|x^{*}\right\|\right)+32 M \bar{c}^{-1} .
$$

Therefore, by Lemma 2.3 and by hypothesis (5.22), we conclude that $\left\|x_{n}-z_{n}\right\| \rightarrow 0$. In addition, by Theorem 3.8,

$$
\left\|x_{n}-\bar{x}^{*}\right\| \leq\left\|x_{n}-z_{n}\right\|+\left\|z_{n}-\bar{x}^{*}\right\| \longrightarrow 0 \quad \text { as } n \longrightarrow \infty,
$$

which implies that $\left\{x_{n}\right\}$ converges strongly to $\bar{x}^{*}$.

5.2. In this subsection, we study an iterative regularization method for (3.1) with a perturbed operator and perturbed right-hand side. Assume that, instead of $f$ and $A$, we have the sequences $\left\{f_{n}\right\}, f_{n} \in E$, and $\left\{A_{n}\right\}, A_{n}: D\left(A_{n}\right) \subseteq E \rightarrow E$, such that

$$
\begin{gathered}
\left\|f_{n}-f\right\| \leq \delta_{n}, \\
\left\|A_{n} x-A x\right\| \leq \omega_{n} \zeta(\|x\|)+\mu_{n}, \quad \forall x \in G,
\end{gathered}
$$

where $\zeta(t)$ is a positive and bounded function defined on $\mathbb{R}^{+}, G \subset D\left(A_{n}\right)$ and $G \subset D(A)$. Thus, in reality, the following equations are given:

$$
A_{n} y=f_{n},
$$

which may not have a solution. Consider the regularizing iterative algorithm

$$
y_{n+1}=Q_{G}\left(y_{n}-\epsilon_{n}\left(A_{n} y_{n}+\alpha_{n} y_{n}-f_{n}\right)\right), \quad n=0,1,2, \ldots,
$$

where $Q_{G}$ is a nonexpansive retraction of $E$ onto $G$. 
Theorem 5.3. Assume that all the conditions of Theorems 3.8 and 5.1 are fulfilled. Suppose that there exist sequences of positive numbers $\omega_{n}, \mu_{n}$, and $\delta_{n}$ converging to zero as $n \rightarrow \infty$, such that (5.39) holds. Suppose that

$$
\alpha_{n} \longrightarrow 0, \quad \frac{\omega_{n}+\mu_{n}+\delta_{n}}{\alpha_{n}} \longrightarrow 0, \quad \frac{\rho_{E}\left(\epsilon_{n}\right)}{\epsilon_{n} \alpha_{n}} \longrightarrow 0, \quad \frac{\left|\alpha_{n}-\alpha_{n+1}\right|}{\epsilon_{n} \alpha_{n}^{2}} \longrightarrow 0
$$

as $n \rightarrow \infty$. Starting from arbitrary $y_{0} \in G$ define the sequence $\left\{y_{n}\right\}$ by (5.41). Then there exists $1>d>0$ such that whenever

$$
\epsilon_{n} \leq d, \quad \frac{\rho_{E}\left(\epsilon_{n}\right)}{\epsilon_{n} \alpha_{n}} \leq d^{2}
$$

for all $n \geq 0$, the sequence $\left\{y_{n}\right\}$ is bounded and converges in norm to the solution $\bar{x}^{*}$, where $\bar{x}^{*}$ is the unique solution of inequality (3.9).

Proof. First of all, as in the proof of Theorem 5.1, we aim at showing that $\left\{y_{n}\right\}$ is bounded. To this end, introduce again a closed ball $B_{r}\left(x^{*}\right)$ with sufficiently large radius $r>0$ such that $r \geq 2\left\|x^{*}\right\|$ and $y_{0} \in B_{r}\left(x^{*}\right)$. And construct again the set $S=B_{r}\left(x^{*}\right) \cap G$. Without loss of generality, according to (5.42), put $\omega_{n} \leq \bar{\omega}, \mu_{n} \leq \bar{\mu}, \delta_{n} \leq \bar{\delta}$, and

$$
M:=\frac{3}{2} r+\|f\|+\sup \{\|A y\|: y \in S\}+\bar{\omega} M_{\zeta}+\bar{\mu}+\bar{\delta}
$$

where

$$
M_{\zeta}=\sup \{\zeta(\|y\|): y \in S\}
$$

We claim that $\left\{y_{n}\right\}$ is bounded.

Assume that $y_{n} \in S$ and show that $y_{n+1} \in S$. We denote

$$
p_{n}=y_{n}-\epsilon_{n}\left(A_{n} y_{n}+\alpha_{n} y_{n}-f_{n}\right) \text {. }
$$

The operator $Q_{G}$ is nonexpansive, therefore, by (5.41) we have

$$
\begin{aligned}
\left\|y_{n+1}-x^{*}\right\| \leq & \left\|Q_{G} p_{n}-Q_{G} x^{*}\right\| \\
\leq & \left\|y_{n}-x^{*}-\epsilon_{n}\left(A_{n} y_{n}+\alpha_{n} y_{n}-f_{n}\right)\right\| \\
\leq & \left\|y_{n}-x^{*}\right\|+\epsilon_{n}\left\|A_{n} y_{n}+\alpha_{n} y_{n}-f_{n}\right\| \\
\leq & \left\|y_{n}-x^{*}\right\|+\left\|A y_{n}\right\|+\left\|A_{n} y_{n}-A y_{n}\right\| \\
& +\left\|y_{n}-x^{*}\right\|+\left\|x^{*}\right\|+\|f\|+\left\|f_{n}-f\right\| .
\end{aligned}
$$

Then

$$
\begin{aligned}
\left\|y_{n+1}-x^{*}\right\| & \leq r+\sup _{y \in S}\|A y\|+\omega_{n} \sup _{y \in S} \zeta(\|y\|)+\mu_{n}+r+\frac{1}{2} r+\|f\|+\delta_{n} \\
& \leq r+M=\bar{M}, \\
\left\|y_{n+1}-y_{n}\right\| & =\varepsilon_{n}\left\|A_{n} y_{n}+\alpha_{n} y_{n}-f_{n}\right\| \leq \varepsilon_{n} M .
\end{aligned}
$$


28 Nonlinear Ill-posed problems with accretive operators

Moreover, by (2.3), (2.6), and (5.41) one gets similarly to (5.10) that

$$
\begin{aligned}
\left\|y_{n+1}-x^{*}\right\|^{2} \leq & \left\|y_{n}-x^{*}-\epsilon_{n}\left(A_{n} y_{n}+\alpha_{n} y_{n}-f_{n}\right)\right\|^{2} \\
\leq & \left\|y_{n}-x^{*}\right\|^{2}-2 \epsilon_{n}\left\langle A_{n} y_{n}-f_{n}, J\left(y_{n}-x^{*}\right)\right\rangle \\
& -2 \epsilon_{n} \alpha_{n}\left\langle y_{n}, J\left(y_{n}-x^{*}\right)\right\rangle+16 L C M \bar{M} \rho_{E}\left(\varepsilon_{n}\right) \\
\leq & \left\|y_{n}-x^{*}\right\|^{2}-2 \epsilon_{n}\left\langle A y_{n}-f, J\left(y_{n}-x^{*}\right)\right\rangle \\
& -2 \epsilon_{n}\left\langle A_{n} y_{n}-A y_{n}, J\left(y_{n}-x^{*}\right)\right\rangle-2 \epsilon_{n}\left\langle f_{n}-f, J\left(y_{n}-x^{*}\right)\right\rangle \\
& -2 \epsilon_{n} \alpha_{n}\left\langle y_{n}, J\left(y_{n}-x^{*}\right)\right\rangle+16 L C M \bar{M} \rho_{E}\left(\varepsilon_{n}\right) .
\end{aligned}
$$

Since $x^{*}$ is a solution of the equation $A x=f$ on $G$ and $y_{n} \in G$ for all $n \geq 0$, we can write

$$
\left\langle A y_{n}-f, J\left(y_{n}-x^{*}\right)\right\rangle \geq 0
$$

Then (5.50) implies the inequality

$$
\begin{aligned}
\left\|y_{n+1}-x^{*}\right\|^{2} \leq & \left\|y_{n}-x^{*}\right\|^{2}-2 \epsilon_{n} \alpha_{n}\left\langle y_{n}, J\left(y_{n}-x^{*}\right)\right\rangle \\
& +2 \epsilon_{n}\left(\omega_{n} M_{\zeta}+\mu_{n}+\delta_{n}\right)\left\|y_{n}-x^{*}\right\|+16 \operatorname{LMC} \bar{M} \rho_{E}\left(\varepsilon_{n}\right) .
\end{aligned}
$$

Denoting $D=8 L C M \bar{M}+r M_{\zeta}^{+}$, where $M_{\zeta}^{+}=\max \left\{1, M_{\zeta}\right\}$, we obtain

$$
\left\langle y_{n}, J\left(y_{n}-x^{*}\right)\right\rangle \leq 8 L C M \bar{M} \frac{\rho_{E}\left(\varepsilon_{n}\right)}{\epsilon_{n} \alpha_{n}}+\frac{r\left(\omega_{n} M_{\zeta}+\mu_{n}+\delta_{n}\right)}{\alpha_{n}} \leq D K,
$$

because of the given inequalities

$$
\frac{\rho_{E}\left(\epsilon_{n}\right)}{\alpha_{n} \epsilon_{n}} \leq K=d^{2}, \quad \frac{\omega_{n}+\mu_{n}+\delta_{n}}{\alpha_{n}} \leq K=d^{2}, \quad \forall n \geq 0 .
$$

The rest of the boundedness proof of $\left\{y_{n}\right\}$ follows as in the proof of Theorem 5.1. Thus, there exists $\widetilde{C}$ such that $\left\|y_{n}\right\| \leq \widetilde{C}$.

We present next the convergence analysis of (5.41). By convexity of $\|x\|^{2}$, we obtain as in (5.25) the following:

$$
\begin{aligned}
\left\|y_{n+1}-z_{n+1}\right\|^{2} \leq & \left\|y_{n+1}-z_{n}\right\|^{2}+2\left\langle z_{n+1}-z_{n}, J\left(z_{n+1}-y_{n+1}\right)\right\rangle \\
\leq & \left\|y_{n+1}-z_{n}\right\|^{2}+2\left\langle z_{n+1}-z_{n}, J\left(z_{n}-y_{n+1}\right)\right\rangle \\
& +2\left\langle z_{n+1}-z_{n}, J\left(z_{n+1}-y_{n+1}\right)-J\left(z_{n}-y_{n+1}\right)\right\rangle \\
\leq & \left\|y_{n+1}-z_{n}\right\|^{2}+2\left\|z_{n}-y_{n+1}\right\| \cdot\left\|z_{n+1}-z_{n}\right\| \\
& +\left(16 \bar{c}^{-1}+C_{1}\right) \rho_{E}\left(\left\|z_{n+1}-z_{n}\right\|\right),
\end{aligned}
$$


where $C_{1}$ is defined by (5.29). Moreover,

$$
\begin{aligned}
\left\|y_{n+1}-z_{n}\right\|^{2}= & \left\|Q_{G} p_{n}-Q_{G} z_{n}\right\|^{2} \leq\left\|p_{n}-z_{n}\right\|^{2} \\
\leq & \left\|y_{n}-z_{n}\right\|^{2}+2\left\langle p_{n}-y_{n}, J\left(p_{n}-z_{n}\right)\right\rangle \\
= & \left\|y_{n}-z_{n}\right\|^{2}+2\left\langle p_{n}-y_{n}, J\left(y_{n}-z_{n}\right)\right\rangle \\
& +2\left\langle p_{n}-y_{n}, J\left(p_{n}-z_{n}\right)-J\left(y_{n}-z_{n}\right)\right\rangle \\
= & \left\|y_{n}-z_{n}\right\|^{2}-2 \epsilon_{n}\left\langle A_{n} y_{n}+\alpha_{n} y_{n}-f_{n}, J\left(y_{n}-z_{n}\right)\right\rangle \\
& +2\left\langle p_{n}-y_{n}, J\left(p_{n}-z_{n}\right)-J\left(y_{n}-z_{n}\right)\right\rangle \\
\leq & \left\|y_{n}-z_{n}\right\|^{2}-2 \epsilon_{n}\left\langle A z_{n}+\alpha_{n} z_{n}-f, J\left(y_{n}-z_{n}\right)\right\rangle \\
& +2 \epsilon_{n}\left(\left\|A_{n} y_{n}-A y_{n}\right\|+\left\|f_{n}-f\right\|\right)\left\|y_{n}-z_{n}\right\|-2 \epsilon_{n} \alpha_{n}\left\|y_{n}-z_{n}\right\|^{2} \\
& +2\left\langle p_{n}-y_{n}, J\left(p_{n}-z_{n}\right)-J\left(y_{n}-z_{n}\right)\right\rangle .
\end{aligned}
$$

Now (5.39), (5.46), and (5.56) yield

$$
\begin{aligned}
\left\|y_{n+1}-z_{n}\right\|^{2} \leq & \left\|y_{n}-z_{n}\right\|^{2}-2 \epsilon_{n} \alpha_{n}\left\|y_{n}-z_{n}\right\|^{2}+2 \epsilon_{n}\left\|y_{n}-z_{n}\right\|\left(\omega_{n} \zeta\left(\left\|y_{n}\right\|\right)+\mu_{n}+\delta_{n}\right) \\
& +2\left\langle p_{n}-y_{n}, J\left(p_{n}-z_{n}\right)-J\left(y_{n}-z_{n}\right)\right\rangle \\
\leq & \left\|y_{n}-z_{n}\right\|^{2}-2 \epsilon_{n} \alpha_{n}\left\|y_{n}-z_{n}\right\|^{2}+2 \epsilon_{n}\left\|y_{n}-z_{n}\right\|\left(\omega_{n} \zeta\left(\left\|y_{n}\right\|\right)+\mu_{n}+\delta_{n}\right) \\
& +16\left\|p_{n}-y_{n}\right\|^{2}+C_{4}(n) \rho_{E}\left(\left\|p_{n}-y_{n}\right\|\right),
\end{aligned}
$$

where

$$
C_{4}(n)=8 \max \left\{2 L,\left\|z_{n}-y_{n}\right\|+\left\|z_{n}-p_{n}\right\|\right\}
$$

Since $\left\{y_{n}\right\}$ and $\left\{z_{n}\right\}$ are bounded and $A$ is a bounded operator, there exists a constant $d_{1}>0$ such that

$$
\begin{gathered}
\left\|p_{n}-y_{n}\right\|=\epsilon_{n}\left\|A_{n} y_{n}+\alpha_{n} y_{n}-f_{n}\right\| \leq \epsilon_{n} d_{1}, \\
C_{4}(n) \leq 8 \max \left\{2 L, \widetilde{C}+M+2\left\|x^{*}\right\|\right\}=C_{4} .
\end{gathered}
$$

Then

$$
16 \epsilon_{n}^{2} d_{1}^{2}+C_{4}(n) \rho_{E}\left(\epsilon_{n} d_{1}\right) \leq C_{5} \rho_{E}\left(\epsilon_{n} d_{1}\right)
$$

where

$$
C_{5}=\left(16 \bar{d}^{-1}+C_{4}\right), \quad \bar{d}=\left(\sqrt{1+d_{1}^{2}}+1\right)^{-1} .
$$


Substituting (5.57) for (5.55), we deduce that

$$
\begin{aligned}
\left\|y_{n+1}-z_{n+1}\right\|^{2} \leq & \left(1-2 \epsilon_{n} \alpha_{n}\right)\left\|y_{n}-z_{n}\right\|^{2}+2 \epsilon_{n}\left\|y_{n}-z_{n}\right\|\left(\omega_{n} \zeta\left(\left\|y_{n}\right\|\right)+\delta_{n}+\mu_{n}\right) \\
& +C_{5} \rho_{E}\left(\epsilon_{n} d_{1}\right)+C_{6} \rho_{E}\left(\left\|z_{n+1}-z_{n}\right\|\right) \\
& +2\left\|z_{n}-y_{n+1}\right\| \cdot\left\|z_{n+1}-z_{n}\right\|,
\end{aligned}
$$

where $C_{6}=16 \bar{c}^{-1}+C_{1}$. Finally, there exists $C>0$ such that $\left\|y_{n}-z_{n}\right\| \leq C$ and then

$$
\begin{aligned}
\left\|y_{n+1}-z_{n+1}\right\|^{2} \leq & \left\|y_{n}-z_{n}\right\|^{2}-2 \epsilon_{n} \alpha_{n}\left\|y_{n}-z_{n}\right\|^{2}+2 \epsilon_{n} C\left(\omega_{n} \zeta(\widetilde{C})+\delta_{n}+\mu_{n}\right) \\
& +C_{5} \rho_{E}\left(\epsilon_{n} d_{1}\right)+2\left\|x^{*}\right\|\left(C_{6}+C\right) \frac{\left|\alpha_{n}-\alpha_{n+1}\right|}{\alpha_{n}},
\end{aligned}
$$

because $\rho_{E}(\tau) \leq \tau$. Now, the conclusion $\left\|y_{n}-z_{n}\right\| \rightarrow 0$ follows from Lemma 2.3. By Theorem 3.8,

$$
\left\|y_{n}-x^{*}\right\| \leq\left\|y_{n}-z_{n}\right\|+\left\|z_{n}-x^{*}\right\| \longrightarrow 0 \quad \text { as } n \longrightarrow \infty \text {. }
$$

Thus, $\left\{y_{n}\right\}$ converges strongly to $x^{*}$. The proof is accomplished.

5.3. Next we study the iterative regularization method for (3.1) defined by

$$
w_{n+1}:=Q_{G_{n+1}}\left(w_{n}-\epsilon_{n}\left(A w_{n}+\alpha_{n} w_{n}-f\right)\right), \quad n=0,1,2, \ldots,
$$

on approximately given sets $G_{n}$, where, for each $n, Q_{G_{n}}$ is a sunny nonexpansive retraction of $E$ onto $G_{n}$.

Theorem 5.4. Assume that all the conditions of Theorems 3.8 and 5.1 are fulfilled and $G_{n} \subseteq$ $\operatorname{int} D(A), n=1,2, \ldots$, are closed convex sets such that the Hausdorff distance $\mathscr{H}_{E}\left(G_{n}, G\right) \leq$ $\sigma_{n} \leq \bar{\sigma}, \sigma_{n+1} \leq \sigma_{n}$. Denote $\bar{G}=G \cup \widetilde{G}$, where $\widetilde{G}=\bigcup G_{n}$. Assume that an operator $A: \bar{G} \rightarrow E$ is accretive and bounded. Assume that conditions (5.22) hold and that

$$
\frac{\sqrt{h_{E}\left(\sigma_{n}\right)}}{\epsilon_{n} \alpha_{n}} \longrightarrow 0 \text { as } n \longrightarrow \infty \text {. }
$$

If the iterative sequence $\left\{w_{n}\right\}$ generated by (5.65) is bounded, then it converges strongly to $\bar{x}^{*}$, where $\bar{x}^{*}$ is the unique solution of inequality (3.9).

Proof. Denote

$$
Z x=x-\epsilon_{n}\left(A x+\alpha_{n} x-f\right) .
$$

Since $\left\{w_{n}\right\}$ is bounded and, hence, $\left\{A w_{n}\right\}$ is also bounded, then there exists a constant $d>0$ such that

$$
\left\|Z w_{n}\right\|=\left\|w_{n}-\epsilon_{n}\left(A w_{n}+\alpha_{n} w_{n}-f\right)\right\| \leq d .
$$

By analogy,

$$
\left\|Z x_{n}\right\|=\left\|x_{n}-\epsilon_{n}\left(A x_{n}+\alpha_{n} x_{n}-f\right)\right\| \leq d,
$$


Ya. I. Alber et al. 31

where $\left\{x_{n}\right\}$ is the bounded sequence generated by (5.1) (see Theorem 5.2). From (5.65) and (5.1), we have

$$
\begin{aligned}
\left\|w_{n+1}-x_{n+1}\right\| & =\left\|Q_{G_{n+1}} Z w_{n}-Q_{G} Z x_{n}\right\| \\
& \leq\left\|Q_{G} Z w_{n}-Q_{G} Z x_{n}\right\|+\left\|Q_{G_{n+1}} Z w_{n}-Q_{G} Z w_{n}\right\| .
\end{aligned}
$$

Estimate the first term of the right-hand side of the previous inequality:

$$
\begin{aligned}
\left\|Q_{G} Z w_{n}-Q_{G} Z x_{n}\right\|^{2} \leq\left\|Z w_{n}-Z x_{n}\right\|^{2} \\
\leq\left\|w_{n}-x_{n}\right\|^{2}-2 \epsilon_{n}\left\langle A w_{n}-A x_{n}+\alpha_{n}\left(w_{n}-x_{n}\right), J\left(Z w_{n}-Z x_{n}\right)\right\rangle \\
\leq\left\|w_{n}-x_{n}\right\|^{2}-2 \epsilon_{n} \alpha_{n}\left\|w_{n}-x_{n}\right\|^{2}-2 \epsilon_{n}\left\langle A w_{n}-A x_{n}, J\left(w_{n}-x_{n}\right)\right\rangle \\
\quad-2 \epsilon_{n}\left\langle A w_{n}-A x_{n}+\alpha_{n}\left(w_{n}-x_{n}\right), J\left(Z w_{n}-Z x_{n}\right)-J\left(w_{n}-x_{n}\right)\right\rangle \\
\leq\left\|w_{n}-x_{n}\right\|^{2}-2 \epsilon_{n} \alpha_{n}\left\|w_{n}-x_{n}\right\|^{2}+16 \epsilon_{n}^{2}\left\|A w_{n}-A x_{n}+\alpha_{n}\left(w_{n}-x_{n}\right)\right\|^{2} \\
\quad+C_{7}(n) \rho_{E}\left(\epsilon_{n}\left\|A w_{n}-A x_{n}+\alpha_{n}\left(w_{n}-x_{n}\right)\right\|\right),
\end{aligned}
$$

where

$$
C_{7}(n)=8 \max \left\{2 L,\left\|Z w_{n}-Z x_{n}\right\|+\left\|w_{n}-x_{n}\right\|\right\} .
$$

Thus, there exists a constant $C_{7}>0$ such that $C_{7}(n) \leq C_{7}$.

Using Lemma 2.7, we come to the following inequality:

$$
\left\|Q_{G_{n+1}} Z w_{n}-Q_{G} Z w_{n}\right\| \leq 16(R+\bar{\sigma})(2 d+q) h_{B}\left(8 q^{-1} \sigma_{n+1}\right),
$$

where $R=2(2 d+q), q=\max \left\{q_{1}, q_{2}\right\}, q_{1}=\operatorname{dist}\left(\theta, G_{1}\right), q_{2}=\max \left\{\operatorname{dist}\left(\theta, G_{n}\right)\right\}, n=0,1$, $2, \ldots$, and $\theta$ is the origin of the Banach space $E$. Hence, from (5.70), (5.71), and (5.73) we get

$$
\begin{aligned}
\left\|w_{n+1}-x_{n+1}\right\|^{2} \leq & \left\|w_{n}-x_{n}\right\|^{2}-2 \epsilon_{n} \alpha_{n}\left\|w_{n}-x_{n}\right\|^{2}+16 \epsilon_{n}^{2} C+C_{7} \rho_{E}\left(\epsilon_{n} C\right) \\
& +\left(\bar{C} h_{B}\left(8 q^{-1} \sigma_{n+1}\right)\right)^{1 / 2}
\end{aligned}
$$

for $\bar{C}=16(R+\bar{\sigma})(2 d+q)$ and some $C>0$.

Since (5.66) holds, we conclude that $\left\|w_{n}-x_{n}\right\| \rightarrow 0$ as $n \rightarrow \infty$. Thus, for all $n \geq 0$, one has

$$
\left\|w_{n+1}-\bar{x}^{*}\right\| \leq\left\|w_{n+1}-x_{n+1}\right\|+\left\|x_{n+1}-\bar{x}^{*}\right\|,
$$

and, therefore,

$$
\left\|w_{n}-\bar{x}^{*}\right\| \longrightarrow 0 \text { as } n \longrightarrow \infty \text {. }
$$

The proof is complete. 
Remark 5.5. Obviously, if $G_{n}$ are bounded, then all $w_{n}$ are bounded too.

Now we are able to combine Theorems 5.2, 5.3, and 5.4 in order to investigate the iterative regularization method for (3.1) with perturbed data $A, f$, and $G$ defined by the following algorithm:

$$
u_{n+1}:=Q_{G_{n+1}}\left(u_{n}-\epsilon_{n}\left(A_{n} u_{n}+\alpha_{n} u_{n}-f_{n}\right)\right), \quad n=0,1,2, \ldots
$$

Theorem 5.6. Suppose that the conditions of Theorems 5.2, 5.3, and 5.4 are fulfilled. If the iterative sequence $\left\{w_{n}\right\}$ generated by (5.77) is bounded, then it converges strongly to $\bar{x}^{*}$, where $\bar{x}^{*}$ is the unique solution of inequality (3.9).

\section{Acknowledgments}

The first author thanks The Abdus Salam International Centre for Theoretical Physics (ICTP), Trieste, Italy, where he was a Visiting Professor while this paper was written. This work was supported in part by the Kamea Program of the Ministry of Absorption.

\section{References}

[1] Ya. I. Alber, On the solution of equations and variational inequalities with maximal monotone operators, Soviet Math. Dokl. 20 (1979), no. 4, 871-876.

[2] _ Metric and generalized projection operators in Banach spaces: Properties and applications, Theory and Applications of Nonlinear Operators of Accretive and Monotone Type (A. Kartsatos, ed.), Lecture Notes in Pure and Appl. Math., vol. 178, Dekker, New York, 1996, pp. 15-50.

[3] Ya. I. Alber, A. G. Kartsatos, and E Litsyn, Iterative solution of unstable variational inequalities on approximately given sets, Abstr. Appl. Anal. 1 (1996), no. 1, 45-64.

[4] Ya. I. Alber and A. I. Notik, Geometric properties of Banach spaces and approximate methods for solving nonlinear operator equations, Soviet Math. Dokl. 29 (1984), no. 3, 611-616.

[5] _ Parallelogram inequalities in Banach spaces and some properties of a duality mapping, Ukrainian Math. J. 40 (1988), no. 6, 650-652.

[6] Ya. I. Alber, S. Reich, and I. Ryazantseva, Nonlinear problems with accretive and d-accretive mappings, preprint, 2003.

[7] Ya. I. Alber, S. Reich, and J.-C. Yao, Iterative methods for solving fixed-point problems with nonself-mappings in Banach spaces, Abstr. Appl. Anal. 2003 (2003), no. 4, 193-216.

[8] Ya. I. Alber and I. Ryazantseva, Nonlinear Ill-posed problems of monotone type, Tech. Rep., Technion, Haifa, 2003.

[9] Ya. I. Alber and J.-C. Yao, Algorithm for generalized multi-valued co-variational inequalities in Banach spaces, Funct. Differ. Equ. 7 (2000), no. 1-2, 5-13 (2001).

[10] F. E. Browder, Nonlinear functional analysis and nonlinear partial differential equations, Differential Equations and Their Applications, Bratislava, 1967, pp. 89-113.

[11] Nonlinear mappings of nonexpansive and accretive type in Banach spaces., Bull. Amer. Math. Soc. 73 (1967), 875-882.

[12] Nonlinear operators and nonlinear equations of evolution in Banach spaces, Nonlinear Functional Analysis (Proc. Sympos. Pure Math., Vol. XVIII, Part 2, Chicago, Ill., 1968), American Mathematical Society, Rhode Island, 1976, pp. 1-308.

[13] C. E. Chidume, H. Zegeye, and B. Ntatin, A generalized steepest descent approximation for the zeros of m-accretive operators, J. Math. Anal. Appl. 236 (1999), no. 1, 48-73. 
[14] I. Cioranescu, Geometry of Banach Spaces, Duality Mappings and Nonlinear Problems, Mathematics and Its Applications, vol. 62, Kluwer Academic Publishers, Dordrecht, 1990.

[15] L. Deng, Iteration processes for nonlinear Lipschitzian strongly accretive mappings in $L_{p}$-spaces, J. Math. Anal. Appl. 188 (1994), no. 1, 128-140.

[16] J. Diestel, Geometry of Banach Spaces—Selected Topics, Lecture Notes in Mathematics, vol. 485, Springer-Verlag, Berlin, 1975.

[17] W. G. Dotson, On the Mann iterative process, Trans. Amer. Math. Soc. 149 (1970), 65-73.

[18] T. Figiel, On the moduli of convexity and smoothness, Studia Math. 56 (1976), no. 2, 121-155.

[19] K. Goebel and S. Reich, Uniform Convexity, Hyperbolic Geometry, and Nonexpansive Mappings, Monographs and Textbooks in Pure and Applied Mathematics, vol. 83, Marcel Dekker, New York, 1984.

[20] S. Ishikawa, Fixed points by a new iteration method, Proc. Amer. Math. Soc. 44 (1974), no. 1, 147-150.

[21] J. Lindenstrauss and L. Tzafriri, Classical Banach Spaces. II. Function Spaces, Ergebnisse der Mathematik und ihrer Grenzgebiete, vol. 97, Springer, Berlin, 1979.

[22] W. R. Mann, Mean value methods in iteration, Proc. Amer. Math. Soc. 4 (1953), 506-510.

[23] R. H. Martin Jr., A global existence theorem for autonomous differential equations in a Banach space, Proc. Amer. Math. Soc. 26 (1970), 307-314.

[24] I. Şerb, Estimates for the modulus of smoothness and convexity of a Banach space, Mathematica (Cluj) 34(57) (1992), no. 1, 61-70.

[25] M. M. Vainberg, Variational Method and Method of Monotone Operators in the Theory of Nonlinear Equations, Halsted Press (A division of John Wiley \& Sons), New York, 1973.

[26] E. Zeidler, Nonlinear Functional Analysis and Its Applications.-II. Monotone Operators, Springer-Verlag, Berlin, 1985.

Ya. I. Alber: Department of Mathematics, Technion - Israel Institute of Technology, 32000 Haifa, Israel

E-mail address: alberya@math.technion.ac.il

C. E. Chidume: Mathematics Section, The Abdus Salam International Centre for Theoretical Physics, 340314 Trieste, Italy

E-mail address: chidume@ictp.it

H. Zegeye: Mathematics Section, The Abdus Salam International Centre for Theoretical Physics, 34014 Trieste, Italy

E-mail address: habz@ictp.it 